\section{Non-Line-of-Sight Mobile Positioning Using Factor Graphs}

\author{
Hongqing Liu, Frankie Kit Wing Chan, and Hing Cheung So
}

\begin{abstract}
Utilizing time-of-arrival measurements, we propose a recursive algorithm for estimating the location of a mobile station in a nonline-of-sight environment. By formulating the problem in a factor graph framework, messages are iteratively passed to obtain the mobile station position. Numerical results show that the proposed algorithm is more computationally attractive or reliable than existing methods and is able to achieve performance very close to the optimum benchmarks.
\end{abstract}

Index Terms-Factor graph, mobile positioning, non-line-of-sight.

\section{INTRODUCTION}

Mobile station positioning has received significant attention since the Federal Communications Commission [1] in the U.S. adopted rules to improve Enhanced 911 services [2]. It turns out that mobile information also has many other applications, e.g., tracking people who need constant attention such as the mentally impaired and young children, intelligent transport systems, location billing, and interactive map consultation. One mobile location solution is achieved by installing a global positioning system receiver at the mobile station. On the other hand, a wireless location system is a more cost-effective solution since base stations in existing wireless networks can be employed for mobile position estimation. Conventional wireless positioning approaches are based on the time of arrival, received signal strength, time difference of arrival, and/or angle-of-arrival measurements received from several base stations with known positions, which have nonlinear relationships with the mobile station position [3]. Once the measurements are available, the mobile position can be estimated by solving the corresponding nonlinear equations by directly using the nonlinear leastsquares technique [4]. Nevertheless, there is no guarantee on global convergence, unless the initial guess is sufficiently close to the global minimum. On the other hand, the linear least-squares methodology provides a global solution and solves the problem by linearizing the nonlinear equations via the introduction of an extra variable [5]. Based on factor graphs, a mobile station positioning algorithm is proposed in [6] and [7], where soft information is efficiently exchanged among local processing units to iteratively estimate the mobile position. All these methods work well in the presence of line-of-sight propagation. However, in practice, due to building blockage, complex terrain, etc., a line-of-sight environment cannot be guaranteed, and it is more reasonable to consider the possibility of non-line-of-sight propagation. It is well known that non-line-of-sight propagation will cause a large positive error in the distance measurements and result in biased position estimates. As a result, the non-line-of-sight effect must be taken into consideration in order to obtain accurate mobile station-location

Manuscript received January 20, 2009; revised May 27, 2009 and July 21, 2009. First published August 11, 2009; current version published November 11, 2009. This work was supported by the Research Grants Council of the Hong Kong Special Administrative Region, China, under Project CityU 119606. The review of this paper was coordinated by Prof. A. M. Tonello.

The authors are with the Department of Electronic Engineering, City University of Hong Kong, Kowloon, Hong Kong (e-mail: hqliuster@gmail.com; ckwf@hkexperts.com; hcso@ee.cityu.edu.hk).

Color versions of one or more of the figures in this paper are available online at http://ieeexplore.ieee.org.

Digital Object Identifier 10.1109/TVT.2009.2029553 estimation. In the literature, there are several schemes [8]-[13] that deal with the non-line-of-sight problem. One approach is to identify the non-line-of-sight propagation, which includes determination by a time-history-based hypothesis test [8], a probabilistic model [9], residual information [10], and maximum-likelihood detection [11]. Once the identification procedure is accomplished, only the line-ofsight measurements are utilized for localization. Apart from incorrect identification, it wastes non-line-of-sight observations, which may help improve the positioning performance. Another standard technique is to use all line-of-sight and non-line-of-sight measurements but with different weights to reduce the non-line-of-sight effect. The weights are determined from the base station layout [12] or from the residuals of an individual base station [13]. Nevertheless, those methods may provide unreliable results as the non-line-of-sight errors cannot completely be eliminated.

In this paper, a recursive Bayesian localization algorithm based on factor graphs is developed for non-line-of-sight mobile station position estimation. Although we take an approach similar to those in [6] and [7], our contributions include the following: 1) The proposed algorithm tackles the more challenging non-line-of-sight environment, and 2) we represent the message by histogram, instead of a single Gaussian approximation, to improve the location performance. The rest of this paper is organized as follows: The problem of mobile station localization in the presence of non-line-of-sight propagation is formulated in Section II. In Section III, algorithm development using the factor graph approach is provided. Simulation results, which contain the comparative performance of the proposed scheme with that of existing methods in terms of localization accuracy and computational complexity, are presented in Section IV. Finally, conclusions are drawn in Section V.

\section{Problem Formulation}

We assume that the mobile station and base stations lie on a 2-D plane, although extension to the 3-D scenario is straightforward. Let $(x, y)$ and $\left(x_{i}, y_{i}\right), i=1,2, \ldots, M$ be the coordinates of the mobile station and base stations, respectively, where $M$ is the number of base stations. In the absence of measurement error, the time of arrival between the mobile station and the $i$ th base station, which is denoted by $t_{i}$, is

$$
t_{i}=\frac{d_{i}}{c}
$$

where $d_{i}=\sqrt{\left(x_{i}-x\right)^{2}+\left(y_{i}-y\right)^{2}}$ and $c$ are the corresponding distance and speed of light, respectively. In our study, the distance measurement, which is denoted by $r_{i}$, is modeled as [10]

$$
r_{i}=d_{i}+q_{i}, \quad i=1,2, \ldots, M
$$

where

$$
q_{i}= \begin{cases}q_{i}^{N}, & \text { with probability } 1-\alpha \\ q_{i}^{N}+q_{i}^{U}, & \text { with probability } \alpha\end{cases}
$$

with $q_{i}^{N} \sim \mathcal{N}\left(0, \sigma^{2}\right)$ and $q_{i}^{U} \sim \mathcal{U}(0, D)$. Here, we assume that the distance measurement noise $q_{i}$ is statistically independent. Furthermore, $q_{i}^{N}$ and $q_{i}^{N}+q_{i}^{U}$ denote the line-of-sight and non-line-ofsight distance measurement errors. Here, Gaussian and uniform 
distributions are denoted by $\mathcal{N}(\cdot)$ and $\mathcal{U}(\cdot)$, respectively. Note that, although we adopt the two most popular probability-density functions for the line-of-sight and non-line-of-sight models, other probability density functions such as exponential plus Gaussian distribution [14] and purely uniform distribution [15] are also applicable to our proposed algorithm. In addition, $\alpha, \sigma^{2}$, and $D$, which represent the probability of non-line-of-sight propagation, the noise power of the lineof-sight distance errors, and the maximum non-line-of-sight distance error, respectively, are known a priori as this information can be determined from field tests. Note that, for ease of presentation, $\alpha, \sigma^{2}$, and $D$ are assumed to be identical among the $M$ base stations. The task is to estimate $x$ and $y$, given $\left\{r_{i}\right\},\left(x_{i}, y_{i}\right), i=1,2, \ldots, M, \sigma^{2}, \alpha$, and $D$.

\section{Algorithm DeVelopment}

\section{A. Review of Factor Graph}

Recently, much attention has been paid to factor graphs since they are able to unify a number of approaches in coding, signal processing, machine learning, statistics, and statistical physics [16], [17]. Factor graphs belong to the family of graphical models, which, in general, represent dependencies among variables by a graph. In particular, a large number of approaches for detection and estimation problems in signal processing, e.g., hidden Markov models and Kalman filtering, can be derived as sum/max-product propagation algorithms in a factor graph. We now review some basics of factor graphs, which are essential for our algorithm development. A more detailed introduction to factor graphs is given in [16] and [17].

Let $\phi=\left\{\phi_{1}, \phi_{2}, \ldots, \phi_{N}\right\}$ be a set of variables and $p(\phi)$, which can be factorized as $\prod_{s} p_{s}\left(\phi_{s}\right)$, where $\phi_{s} \subset \phi$ and $\bigcup_{s} \phi_{s}=\phi$, be the joint probability density function of $\phi$. It is a bipartite graph that expresses this factorization structure. A factor graph has a variable node for each variable and a factor node for each function $p_{s}\left(\phi_{s}\right)$. Furthermore, variable nodes are connected to factor nodes by edges to indicate their probabilistic relation.

In factor graph iteration, messages are sent between variable and factor nodes. The message from the factor node $F_{i}$ to the variable node $\phi_{j}$, which is denoted by $m_{F_{i} \rightarrow \phi_{j}}\left(\phi_{j}\right)$, is the probability of $\phi_{j}$ in different states based on the function $p_{i}$. On the other hand, the message from the variable node $\phi_{j}$ to the factor node $F_{i}$, which is denoted by $m_{\phi_{j} \rightarrow F_{i}}\left(\phi_{j}\right)$, is the probability of node $\phi_{j}$ in different states, based on all the information that node $\phi_{j}$ has, except for that based on the function $p_{i}$. Mathematically, the recursive message update rules are given as [16]

$$
\begin{aligned}
& m_{F_{i} \rightarrow \phi_{j}}\left(\phi_{j}\right)=\int F_{i}\left(\phi_{i}\right) \prod_{l \in \mathcal{N}\left(F_{i}\right) \backslash j} m_{l \rightarrow F_{i}}\left(\phi_{l}\right) d \phi_{i \backslash j} \\
& m_{\phi_{j} \rightarrow F_{i}}\left(\phi_{j}\right)=\prod_{F_{l} \in \mathcal{N}(j) \backslash F_{i}} m_{F_{l} \rightarrow j}\left(\phi_{j}\right)
\end{aligned}
$$

where $\mathcal{N}(i) \backslash j$ denotes all the nodes that are neighbors of node $i$, except for node $j$, and $\phi_{i \backslash j}=\left\{\phi_{i} \backslash \phi_{j}\right\}$ indicates the set $\phi_{i}$, discarding the element $\phi_{j}$. Upon convergence, the parameter estimate of $\phi_{j}$ is given by $\prod_{F_{l} \in \mathcal{N}(j)} m_{F_{l} \rightarrow j}\left(\phi_{j}\right)$.

\section{B. Proposed Algorithm}

Before proceeding, we need to derive the probability density function of the distance measurements. The posterior distribution of the

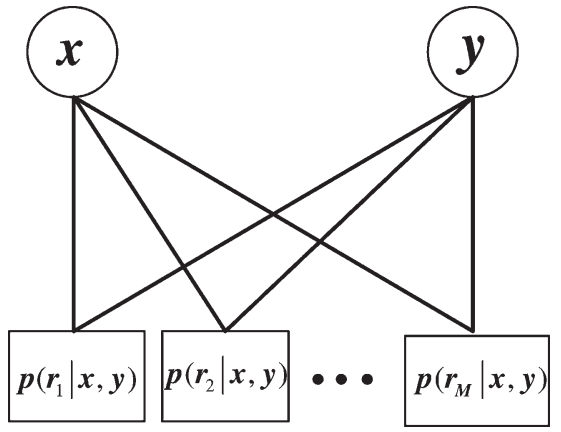

Fig. 1. Factor graph for non-line-of-sight mobile station localization.

mobile location is

$$
\begin{array}{r}
p\left(x, y \mid r_{1}, r_{2}, \ldots, r_{M}\right)=\frac{p\left(r_{1}, r_{2}, \ldots, r_{M} \mid x, y\right) p(x, y)}{p\left(r_{1}, r_{2}, \ldots, r_{M}\right)} \\
\propto p\left(r_{1}, r_{2}, \ldots, r_{M} \mid x, y\right) .
\end{array}
$$

Equation (4) is obtained since the prior $p(x, y)$ is assumed to be uniformly distributed, and $p\left(r_{1}, r_{2}, \ldots, r_{M}\right)$ is constant. Using (1) and the fact that the distance measurements are statistically independent, (4) can be expressed as [10]

$$
p\left(r_{1}, \ldots, r_{M} \mid x, y\right)=\prod_{i=1}^{M} p\left(r_{i} \mid x, y\right)
$$

where

$$
p\left(r_{i} \mid x, y\right)=(1-\alpha) \mathcal{N}\left(r_{i} ; d_{i}, \sigma^{2}\right)+\alpha \mathcal{C}\left(r_{i}, d_{i}, \sigma, D\right)
$$

with $\mathcal{N}\left(r_{i} ; d_{i}, \sigma^{2}\right)$ being the Gaussian probability density function and the random variable, mean, and variance equal to $r_{i}, d_{i}$, and $\sigma^{2}$, respectively. Here, $\mathcal{C}\left(r_{i}, d_{i}, \sigma, D\right)$ is obtained from the convolution of the Gaussian and uniform distributions

$$
\begin{aligned}
\mathcal{C}\left(r_{i}, d_{i}, \sigma, D\right) & =\frac{1}{D} \int_{-\infty}^{\infty}(\mathcal{U}(t)-\mathcal{U}(t-D)) \mathcal{N}\left(r_{i}-t ; d_{i}, \sigma^{2}\right) d t \\
& =\frac{1}{D} \int_{0}^{D} \mathcal{N}\left(r_{i}-t ; d_{i}, \sigma^{2}\right) d t
\end{aligned}
$$

where $\mathcal{U}(\cdot)$ denotes the unit step function. Letting $v=\left(r_{i}-t-\right.$ $\left.d_{i}\right) /(\sqrt{2} \sigma),(6)$ is expressed as

$$
\begin{aligned}
\mathcal{C}\left(r_{i}, d_{i}, \sigma, D\right) & =\frac{1}{D} \int_{\frac{r_{i}-D-d_{i}}{\sqrt{2} \sigma}}^{\substack{r_{i}-d_{i} \\
\sqrt{2} \sigma}} \mathcal{N}(v ; 0,0.5) d v \\
& =\frac{1}{2 D}\left(\operatorname{erf}\left(\frac{r_{i}-d_{i}}{\sqrt{2} \sigma}\right)-\operatorname{erf}\left(\frac{r_{i}-D-d_{i}}{\sqrt{2} \sigma}\right)\right)
\end{aligned}
$$

where $\operatorname{erf}(\cdot)$ is the Gauss error function.

Substituting $F_{i}, \phi_{1}$, and $\phi_{2}$ by $p\left(r_{i} \mid x, y\right), x$, and $y$, respectively, yields the factor graph for non-line-of-sight mobile station position estimation, and its block diagram is shown in Fig. 1. 
In mobile station localization, (2) becomes

$$
\begin{aligned}
& m_{F_{i} \rightarrow x}(x)=\int p\left(r_{i} \mid x, y\right) m_{y \rightarrow F_{i}}(y) d y \\
& m_{F_{i} \rightarrow y}(y)=\int p\left(r_{i} \mid x, y\right) m_{x \rightarrow F_{i}}(x) d x .
\end{aligned}
$$

Applying (3) yields

$$
\begin{aligned}
& m_{x \rightarrow F_{i}}(x)=\prod_{j=1, j \neq i}^{M} m_{F_{j} \rightarrow x}(x)=\frac{\mathcal{M}(x)}{m_{F_{i} \rightarrow x}(x)} \\
& m_{y \rightarrow F_{i}}(y)=\prod_{j=1, j \neq i}^{M} m_{F_{j} \rightarrow y}(y)=\frac{\mathcal{M}(y)}{m_{F_{i} \rightarrow y}(y)}
\end{aligned}
$$

where $\mathcal{M}(x)=\prod_{j=1}^{M} m_{F_{j} \rightarrow x}(x)$, and $\mathcal{M}(y)=\prod_{j=1}^{M} m_{F_{j} \rightarrow y}(y)$. This implies, from (9), that the variable node $x$ can send the message $\prod_{j=1}^{M} m_{F_{j} \rightarrow x}(x)$ to all factor nodes $\left\{F_{i}\right\}$ and let them identify their individual message by division, instead of multiplication, of incoming messages, and the same idea is applicable to (10). Compared with the message multiplication approach, which requires a total of $(M-2) M$ message multiplications, the division approach only needs $(M-1)$ message multiplications and $M$ message divisions. Although division is more complex than multiplication, the division approach is more computationally attractive when $M$ is large. As (7) and (8) have no closed-form solutions, we resort to stochastic integration, i.e., importance sampling, to solve them. In doing so, (7) is approximated as

$$
m_{F_{i} \rightarrow x}(x) \approx \frac{1}{J} \sum_{j=1}^{J} p\left(r_{i} \mid x, y^{(j)}\right)
$$

where $y^{(j)} \sim m_{y \rightarrow F_{i}}(y)$ for sufficiently large $J$. In our algorithm, messages are approximated by a histogram as it facilitates sampling and algebraic operation. We divide the range of $x$ into $R$ equally spacing segments for every $m_{F_{i} \rightarrow x}(x)$, where $R$ is chosen to balance the accuracy and computational complexity. In every segment, a number $x^{(k)}$ is uniformly sampled, and the likelihood of (11) is computed. As a result, the message can be represented by a histogram with the height of every segment equals the normalized likelihood. After that, $\mathcal{M}(x)$ is computed. The values of some bins of $m_{F_{i} \rightarrow x}(x)$ and $m_{F_{i} \rightarrow y}(y)$ may be very small and cause numerical instability during the division in (9) and (10). In this case, the corresponding bins of $\mathcal{M}(x)$ and $\mathcal{M}(y)$ will have even smaller values. For numerical stability and effective sampling, the bins of $\mathcal{M}(x)$, which are less than a certain threshold denoted by $\mu$, are removed. Furthermore, $x_{l}$ and $x_{u}$ are replaced by the lower and upper boundaries of $\mathcal{M}(x)$, respectively. The same procedure is applied to $\mathcal{M}(y), y_{l}$, and $y_{u}$ as well. Next, $m_{x \rightarrow F_{i}}(x)$ and $m_{y \rightarrow F_{i}}(y)$ are computed using (9) and (10), respectively. By recursive message passing, the position estimate is given by $(\mathcal{M}(x), \mathcal{M}(y))$ upon convergence. Our proposed localization algorithm is summarized here.

1) Initialize

$$
\begin{aligned}
m_{x \rightarrow F_{i}}(x)=\frac{\sum_{k=1}^{R} \mathcal{U}\left(x-x_{l}-(k-1) \frac{x_{u}-x_{l}}{R}\right)}{x_{u}-x_{l}} \\
-\frac{\sum_{k=1}^{R} \mathcal{U}\left(x-x_{l}-k \frac{x_{u}-x_{l}}{R}\right)}{x_{u}-x_{l}}
\end{aligned}
$$

for $i=1,2, \ldots, M$. Initialize $m_{y \rightarrow F_{i}}(y)$ in a similar manner. Here, $x_{l}$ and $y_{l}$ are prior lower bounds, and $x_{u}$ and $y_{u}$ are prior upper bounds.

2) Sample $x^{(j)}$ and $y^{(j)}, j=1,2, \ldots, J$ from $m_{x \rightarrow F_{i}}(x)$ and $m_{y \rightarrow F_{i}}(y)$, respectively.

3) Compute

$$
\begin{array}{r}
m_{x \rightarrow F_{i}}(x)=\frac{\sum_{k=1}^{R} m_{F_{i} \rightarrow x}\left(x^{(k)}\right) \mathcal{U}\left(x-x_{l}-(k-1) \frac{x_{u}-x_{l}}{R}\right)}{x_{u}-x_{l}} \\
-\frac{\sum_{k=1}^{R} m_{F_{i} \rightarrow x}\left(x^{(k)}\right) \mathcal{U}\left(x-x_{l}-k \frac{x_{u}-x_{l}}{R}\right)}{x_{u}-x_{l}}
\end{array}
$$

where $m_{F_{i} \rightarrow x}(x)$ is defined in (11), and $x^{(k)} \sim \mathcal{U}(((k-$ 1) $\left.\left.\left(x_{u}-x_{l}\right) / R\right),\left(k\left(x_{u}-x_{l}\right) / R\right)\right)$ for $i=1,2, \ldots, M$. Compute $m_{F_{i} \rightarrow y}(y)$ in a similar fashion.

4) Compute $\mathcal{M}(x)$ and $\mathcal{M}(y)$, and remove those bins whose values are smaller than $\mu$. Update $x_{l}, x_{u}, y_{l}$, and $y_{u}$ as the lower and upper boundaries of $\mathcal{M}(x)$ and $\mathcal{M}(y)$. Compute $m_{x \rightarrow F_{i}}(x)$ in (9) and $m_{y \rightarrow F_{i}}(y)$ in (10), $i=1,2, \ldots, M$.

5) Go back to step 2 until convergence.

\section{Simulation Results}

Computer simulations are conducted to evaluate the performance of the proposed algorithm. The mobile station position is $(1000,2000) \mathrm{m}$. The values of $\alpha, D$, and $J$ are $0.2,1000 \mathrm{~m}$, and 10 , respectively, and $R=10$ and $\mu=10^{-3}$ are assigned. For performance comparison with existing methods, we implement the deterministic approaches Cong-Zhuang [10] and Riba-Urruela [11] algorithms. Both schemes calculate the mobile station positions for all line-of-sight/non-line-ofsight distance measurement combinations, and the one with the lowest error is selected as the position estimate. Note that the former utilizes non-line-of-sight probability and maximum distance error information, whereas the latter does not. Regarding performance benchmarks, we also include the maximum-likelihood estimate of (5) and the Chan-Ho algorithm [5]. The former is realized by Newton's method with initialization using the true position, and the latter assumes to have perfect identification of non-line-of-sight distance measurements and only employs the line-of-sight measurements to estimate the mobile station position. Here, $x_{u}, x_{l}, y_{u}$, and $y_{l}$ are initialized by adding and subtracting $500 \mathrm{~m}$ from the position estimate produced by the Riba-Urruela algorithm. The proposed algorithm is iterated five times, and all simulation results are averages of 500 independent runs. We study the location accuracy using two performance measures: 1) mean square position error, which is the average accuracy in terms of square error, and 2) the distribution of the distance error using cumulative probability density. The empirical mean square position error is computed as $(1 / 500) \sum_{i=1}^{500}\left\{\left(\hat{x}_{i}-x\right)^{2}+\left(\hat{y}_{i}-y\right)^{2}\right\}$, whereas the distance error is $\sqrt{\left\{\left(\hat{x}_{i}-x\right)^{2}+\left(\hat{y}_{i}-y\right)^{2}\right\}}, i=1,2, \ldots, 500$, where $\left(\hat{x}_{i}, \hat{y}_{i}\right)$ is the mobile station position estimate of the $i$ th run.

In the first test, the base stations are fixed at $(0,0),(0,6000)$, $(6000,6000),(6000,0),(6000,-6000),(0,-6000),(-6000,-6000)$, $(-6000,0)$, and $(-6000,6000) \mathrm{m}$. The mean square position errors are plotted against $\sigma^{2}$ in Fig. 2. It is seen that the mean square position errors of the Chan-Ho algorithm are the smallest when $\sigma^{2} \leq$ $50 \mathrm{~dB}$, whereas the maximum-likelihood method performs best when $55 \mathrm{~dB} \leq \sigma^{2} \leq 70 \mathrm{~dB}$. We also observe that the Cong-Zhuang and proposed algorithms have comparable performance, and they are slightly inferior to the maximum-likelihood method. The Riba-Urruela 


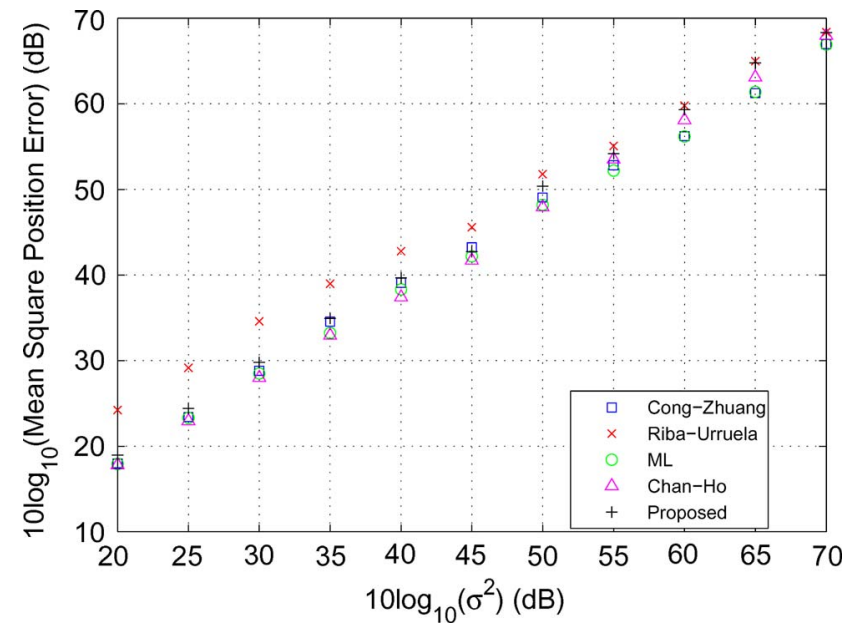

Fig. 2. Mean square position error versus $\sigma^{2}$ in the fixed base-station position case.

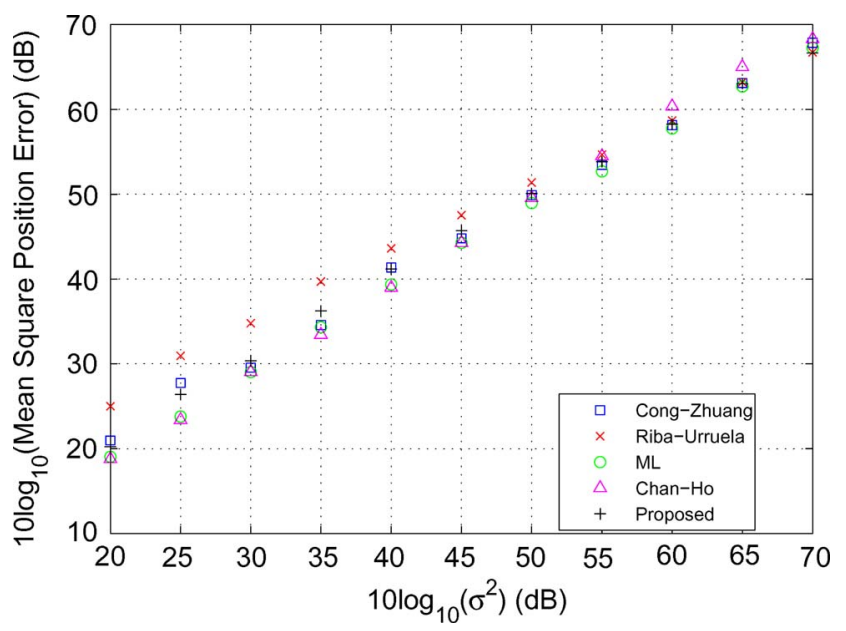

Fig. 3. Mean square position error versus $\sigma^{2}$ in the random base-station position case.

algorithm, which does not utilize prior line-of-sight/non-line-of-sight information, has the worst performance among all methods. It is worthy to point out that, although the Chan-Ho algorithm gives the most accurate position estimation, it can only serve as an ideal reference since perfect line-of-sight/non-line-of-sight detection is impractical.

In the second test, the nine base stations are randomly deployed in each independent run. The mean square position errors are plotted against $\sigma^{2}$ in Fig. 3, and the same conclusion is drawn as in the fixed position case. In both scenarios, the average computational times of the Cong-Zhuang, Riba-Urruela, maximum-likelihood, Chan-Ho, and proposed methods are 0.16, 0.072, 0.02, 0.0005, and $0.088 \mathrm{~s}$, respectively. It shows that the proposed scheme is computationally comparable with the Riba-Urruela algorithm and is less complex than the Cong-Zhuang algorithm.

In Figs. 4 and 5, the cumulative probability densities of the distance errors of different algorithms at $\sigma^{2}=30 \mathrm{~dB}$ for fixed and random base station position cases, respectively, are plotted. It can be observed that the cumulative probability densities of the Cong-Zhuang, maximumlikelihood, and Chan-Ho algorithms are comparable, and they give the best performance. The cumulative probability density of the pro-

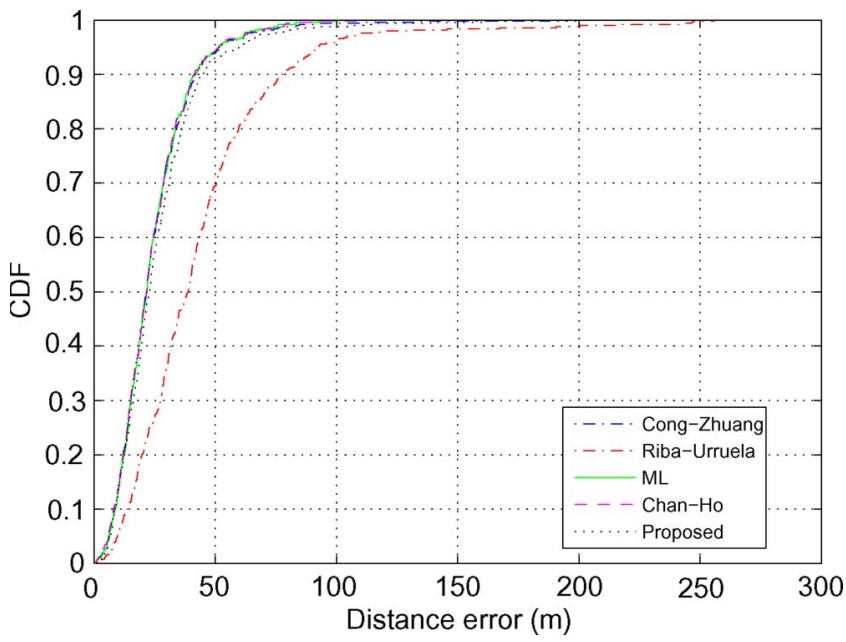

Fig. 4. Cumulative probability density versus distance errors in the fixed basestation position case.

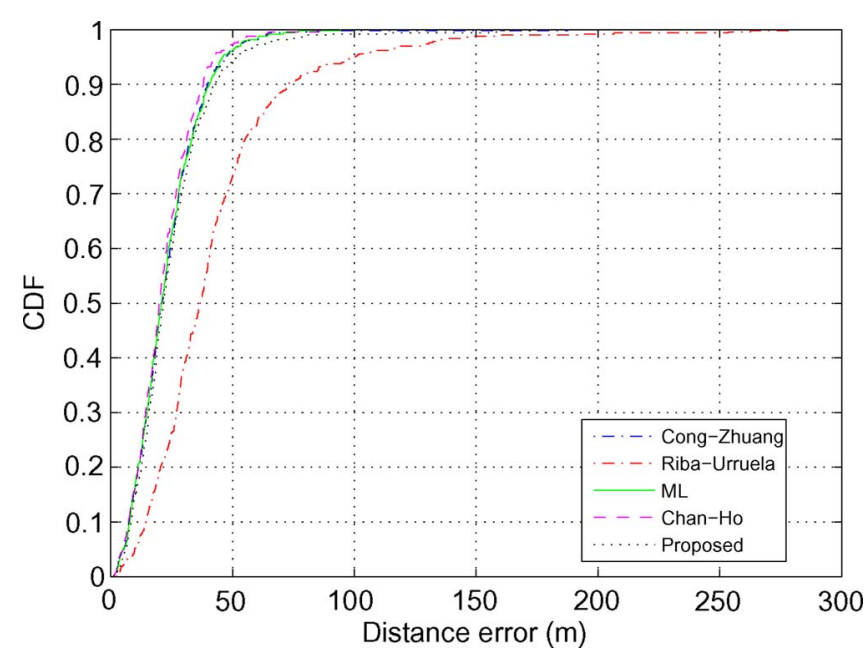

Fig. 5. Cumulative probability density versus distance errors in the random base-station position case.

posed algorithm is very close to the optimum benchmarks, and the Riba-Urruela algorithm performs worst.

In Tables I and II, we tabulate the percentages when the distance error is less than $100 \mathrm{~m}$, which corresponds to the Federal Communications Commission requirement [1], in the fixed and random base station position cases, respectively. Similar to the mean square position error study, the Cong-Zhuang, maximum-likelihood, and Chan-Ho algorithms have the best performance, and the proposed method is slightly inferior to them. On the other hand, the Riba-Urruela algorithm performs the worst.

\section{CONCLUSION}

A recursive algorithm has been devised for mobile positioning in the presence of non-line-of-sight propagation using factor graphs. Simulation results have shown that the proposed algorithm is comparable to the ideal performance benchmarks and is more computationally attractive or accurate than two existing methods. 
TABLE I

Percentage of Distance Errors That Are Less Than 100 m With Fixed Base Station Positions

\begin{tabular}{|c|c|c|c|c|c|}
\hline \multicolumn{1}{|c|}{ Algorithm } & Cong-Zhuang & Riba-Urruela & Maximum Likelihood & Chan-Ho & Proposed \\
\hline 20 & 100 & 100 & 100 & 100 & 100 \\
\hline 25 & 100 & 99.6 & 100 & 100 & 100 \\
\hline 30 & 99.8 & 95 & 100 & 100 & 99.2 \\
\hline 35 & 96.6 & 79.2 & 97.8 & 98 & 95.8 \\
\hline 40 & 77 & 46.6 & 79.4 & 83.6 & 73.6 \\
\hline 45 & 40.4 & 20.4 & 47.6 & 46.2 & 36.4 \\
\hline 50 & 12.2 & 6.4 & 15.2 & 15.4 & 10.2 \\
\hline 55 & 5.8 & 3.2 & 6.2 & 6.6 & 4.2 \\
\hline 60 & 3 & 1.2 & 2.6 & 2 & 1.4 \\
\hline 65 & 1 & 0 & 0.8 & 0.4 & 0.2 \\
\hline 70 & 0 & 0 & 0.8 & 0 & 0 \\
\hline
\end{tabular}

TABLE II

Percentage of Distance Errors That Are Less Than 100 m With Random Base Station Positions

\begin{tabular}{|c|c|c|c|c|c|}
\hline \multicolumn{1}{|c|}{ Algorithm } & Cong-Zhuang & Riba-Urruela & Maximum Likelihood & Chan-Ho & Proposed \\
\hline$\sigma^{2}\left(\mathrm{~m}^{2}\right)$ & 100 & 100 & 100 & 100 & 100 \\
\hline 20 & 100 & 100 & 100 & 100 & 100 \\
\hline 25 & 99.4 & 96.2 & 99.8 & 98.8 & 98.8 \\
\hline 30 & 94.6 & 78.8 & 96.2 & 98 & 94 \\
\hline 40 & 69.2 & 45.8 & 72 & 76 & 67.6 \\
\hline 45 & 34.8 & 22.4 & 35.4 & 40.2 & 35.2 \\
\hline 50 & 9.6 & 8 & 11 & 11.2 & 11 \\
\hline 55 & 6 & 3 & 7 & 3 & 4 \\
\hline 60 & 4.8 & 2 & 4 & 2 & 4 \\
\hline 65 & 2 & 2 & 3 & 1.6 & 1.6 \\
\hline 70 & 0.4 & 0 & 0.16 & 0.4 & 0.4 \\
\hline
\end{tabular}

\section{REFERENCES}

[1] FCC Wireless 911 Requirements Fact Sheet. WTB Policy, Jan. 2001. [Online]. Available: http://www.fcc.gov/pshs/services/911-services/ enhanced911/archives/factsheet_requirements_012001.pdf

[2] RM-8143, CC Docket no. 94-102, Revision of the Commissions Rules to Ensure Compatibility With Enhanced 911 Emergency Calling Systems, Jul. 26, 1999.

[3] J. Caffery, Jr., Wireless Location in CDMA Cellular Radio Systems. Boston, MA: Kluwer, 1990.

[4] J. Caffery, Jr. and G. L. Stüber, "Subscriber location in CDMA cellular networks," IEEE Trans. Veh. Technol., vol. 47, no. 2, pp. 406-416, May 1998

[5] Y. T. Chan and K. C. Ho, "A simple and efficient estimator for hyperbolic location," IEEE Trans. Signal Process., vol. 42, no. 8, pp. 1905-1915, Aug. 1994.

[6] J.-C. Chen, C.-S. Maa, Y.-C. Wang, and J.-T. Chen, "Mobile position location using factor graphs," IEEE Commun. Lett., vol. 7, no. 9, pp. 431433, Sep. 2003.

[7] J.-C. Chen, Y.-C. Wang, C.-S. Maa, and J.-T. Chen, "Network-side mobile position location using factor graphs," IEEE Trans. Wireless Commun., vol. 5, no. 10, pp. 2696-2704, Oct. 2006.

[8] M. P. Wylie and J. Holtzman, "The non-line-of-sight problem in mobile location estimation," in Proc. IEEE Int. Conf. Universal Pers. Commun., Cambridge, U.K., Sep. 1996, vol. 2, pp. 827-831.

[9] J. Borras, P. Hatrack, and N. B. Mandayam, "Decision theoretic framework for NLOS identification," in Proc. IEEE Veh. Technol. Conf., Ottawa, ON, Canada, Nov. 1998, vol. 2, pp. 1583-1587.

[10] L. Cong and W. Zhuang, "Nonline-of-sight error mitigation in mobile location," IEEE Trans. Wireless Commun., vol. 4, no. 2, pp. 560-572, Mar. 2005.

[11] J. Riba and A. Urruela, "A non-line-of-sight mitigation technique based on ML-detection," in Proc. IEEE Int. Conf. Acoust., Speech, Signal Process., Montreal, QC, Canada, May 2004, vol. 2, pp. 153-156.

[12] S. Venkatraman, J. Caffery, Jr., and H. R. You, "Location using LOS range estimation in NLOS environments," in Proc. IEEE Veh. Technol. Conf., Birmingham, AL, May 2002, vol. 2, pp. 856-860.
[13] P. C. Chen, "A non-line-of-sight error mitigation algorithm in location estimation," in Proc. IEEE Wireless Commun. Netw. Conf., New Orleans, LA, Sep. 1999, vol. 1, pp. 316-320.

[14] K. Yu and Y. J. Guo, "Improved positioning algorithms for non-line-ofsight environments," IEEE Trans. Veh. Technol., vol. 57, no. 4, pp. 2342 2353, Jul. 2008.

[15] S. Venkatraman and J. Caffery, Jr., "Hybrid TOA/AOA techniques for mobile location in non-line-of-sight environments," in Proc. IEEE WCNC, Mar. 2004, vol. 1, pp. 274-278.

[16] F. R. Kschischang, B. J. Frey, and H.-A. Loeliger, "Factor graphs and the sum-product algorithm," IEEE Trans. Inf. Theory, vol. 47, no. 2, pp. 498 519, Feb. 2001

[17] H.-A. Loeliger, "An introduction to factor graphs," IEEE Signal Process. Mag., vol. 21, no. 4, pp. 28-41, Jan. 2004. 